\title{
Singing and Its Neuronal Substrates: Evidence from the General Population
}

\author{
Simone Dalla Bella and Magdalena Berkowska
}

\begin{abstract}
This article reviews a range of behavioural and neuroimaging studies with the goal of characterising proficient singing in the general population and identifying its neuronal underpinnings. Evidence is provided to suggest that the majority of individuals can carry a tune, and that a complex neural network engaging motor, auditory and sensorimotor integration areas supports this ability. These mechanisms can be disrupted by a brain insult or neurogenetic disorders leading to inaccurate singing. A variety of poor-singing patterns are described (e.g., with or without concurrent perceptual deficiencies). The study of these poor-singing phenotypes is useful in understanding the complex structure of the song system and its neuronal substrates.
\end{abstract}

Keywords: Singing; Neuroscience; Music; Neuroimaging

\section{Introduction}

Singing is a widespread activity in our society. Most of us, despite not having received musical and vocal training, enjoy the sheer pleasure of singing at parties, with friends, during religious ceremonies and, less often, in solitude. This mostly participatory activity is thought to foster bonding among individuals in group contexts (Mithen, 2006; Wallin et al., 2000), thus fulfilling an important social function. Interestingly, singing is not a trait that is specific to our society. In different cultures, people actively participate in ritual activities through singing, and mothers sing to their babies (Mithen, 2006; Trehub, 2009).

Like uttering the first words and sentences, singing emerges spontaneously, without needing dedicated training or tutoring. From early infancy, individuals display elementary singing abilities. Infants very soon start imitating the vocal characteristics of maternal singing (Trehub \& Trainor, 1999). During the first months of life, they produce vocalisations (e.g., glissandi; Papoušek, 1996) that can be seen as the precursors of music and speech intonation (for a review, see Welch, 2005). This possibility is consistent with recent evidence that the melodic character of this vocalisation may be a predictor of future language development (Wermke et al., 


\section{S. Dalla Bella and M. Berkowska}

2007). The first meaningful vocalisations, including vowels sung at locally stable pitches, emerge by the end of the first year. Yet it is only around 18 months of age that children's vocalisations start to resemble to recognisable songs- that is, mostly short musical phrases repeated over and over (for reviews, see Dowling, 1999; Ostwald, 1973; Welch, 2006). These vocal productions, albeit elementary, exhibit the main characteristics of adult singing (i.e., stable pitch contour and regular beat patterns) except for tonal stability, which is mastered later, around five years of age (Dowling, 1999; Dowling \& Harwood, 1986). At this time, children's vocal productions do not differ qualitatively from adults' singing. The brain circuitries needed for achieving adult proficient singing are already in place.

In this article, we will review experimental evidence regarding singing proficiency (i.e., the ability to sing in-tune and in-time) in the majority-namely in adults without musical/vocal training (referred to hereafter as 'occasional singers'). Voice properties, although relevant for judging whether somebody's singing is 'good' or 'poor' (Himonides \& Welch, 2006), are not taken into account in this article. Results from behavioural and neuroimaging studies will be described with the goal of characterising singing proficiency in the general population, and elucidating its neuronal underpinnings. Particular attention will also be paid to cases of poor singing in otherwise normal individuals (i.e., tone deafness) as a way to understand the mechanisms underlying proficient singing.

\section{Singing Proficiency in the General Population}

Empirical evidence regarding singing proficiency in the general population is scant. This contrasts with the rich literature on the acoustical properties of the singing voice in professional singers (Sundberg, 1987, 1999). This situation may result from the fact that most people who have not received vocal training (i.e., occasional singers) believe that they are inaccurate singers. Such belief is confirmed by occasional singers' assessment of their own sung renditions. For example, almost $60 \%$ of 1,000 university students reported that they cannot imitate melodies (Pfordresher \& Brown, 2007). Moreover, self-declared tone-deaf individuals (around $17 \%$ of the student population) believe that they cannot carry a tune (Cuddy et al., 2005). However, these subjective judgments likely underestimate occasional singers' actual proficiency. Systematic studies based on objective measurements of singing accuracy indicate that the prevalence of poor singing is lower, probably confined to $10-15 \%$ of the population (Dalla Bella \& Berkowska, 2009; Dalla Bella et al., 2007; Pfordresher \& Brown, 2007). We will discuss poor singing in a separate section.

Another reason that may explain why singing proficiency in the general population has been neglected pertains to methodology. Indeed, there is presently no generally accepted standard for the analysis of sung performance. A common method for estimating singing proficiency is to ask peers (e.g., musicians) to assess participants' renditions (Alcock et al., 2000; Hébert et al., 2003; Racette et al., 2006; Schön et al., 2004; Wise \& Sloboda, 2008). This method, albeit time-efficient, has several 
drawbacks. Inconsistencies among raters' judgments are frequent (e.g., Kinsella et al., 1988; Prior et al., 1990). Moreover, peers can hardly provide fine and independent estimates of accuracy on the pitch dimension and on the time dimension. For example, differences of 20-25 cents with respect to the equally tempered scale can go unnoticed even by expert listeners (Vurma \& Ross, 2006). Acoustical methods, based on the extraction of acoustic features from the recordings such as fundamental frequencies and onset times, are valuable alternatives (Dalla Bella et al., 2007; Murayama et al., 2004; Terao et al., 2006). Yet objective and quantitative analysis of singing proficiency (e.g., acoustical analysis) still poses some challenge as compared to recording and analysis of performance on musical instruments (e.g., piano performance).

Early studies on singing proficiency in occasional singers reveal accurate memory for initial pitch and tempo of popular songs (Bergeson \& Trehub, 2002; Halpern, 1989; Levitin, 1994; Levitin \& Cook, 1996). However, occasional singers exhibit low accuracy in pitch-matching tasks. In these tasks, participants without musical training are asked to imitate single pitches; accuracy is computed as the deviation of the produced pitch from the target pitch. Occasional singers deviate by 1.3 semitones on average from the target pitch as compared to 0.5 semitones for musicians (Amir et al., 2003; Murry, 1990; Murry \& Zwiner, 1991; Ternstrom et al., 1988). This finding may lead to the conclusion that occasional singers are indeed very inaccurate in imitating singles pitches. Yet higher accuracy (i.e., below 0.5 semitones) was obtained when pitches to be imitated were synthesised voices or sung productions (Pfordresher \& Brown, 2007; Wise \& Sloboda, 2008).

Two recent studies focused on occasional singers' accuracy in imitating novel melodies. In a study by Pfordresher and Brown (2007) more than 100 university students imitated short melodies with increasing complexity (i.e., a single repeated note, a sequence including a single pitch change and four-note melodies). Most occasional singers successfully imitated melodies (i.e., produced pitches were within \pm 1 semitone from the target pitches). Accuracy was affected by melody complexity (i.e., pitches in the context of melodies were reproduced more poorly compared with sequences including just one interval; for similar results, see Wise \& Sloboda, 2008). In sum, occasional singers are more accurate than previously expected in imitating novel pitch sequences.

In other studies, we examined the accuracy of occasional singers in performing familiar melodies from memory. We adopted an acoustically based method to determine whether participants sang in tune and in time. In a first study (Dalla Bella et al., 2007), occasional singers sang a familiar song with lyrics (the chorus of Gens $d u$ pays, well-known in Quebec). Occasional singers produced pitch intervals less accurately as compared to professional singers, but at the same time sang at a faster tempo. This effect of tempo was examined in a further experiment. Fifteen occasional singers who participated in the previous experiment sang the same melody at a slow tempo. Thirteen of them sang as proficiently as professional singers, in term of both pitch and time accuracy. However, two participants did not improve, and were 


\section{S. Dalla Bella and M. Berkowska}

thereby qualified as 'poor-pitch singers' (see below for a discussion). The positive effect of reducing tempo on singing proficiency was confirmed more recently using a wider selection of melodies and a larger sample (Berkowska \& Dalla Bella, 2009a; Dalla Bella \& Berkowska, 2009).

In sum, occasional singers are not as inaccurate singers as previously believed. The majority can sing in-tune and in-time. Yet, several factors have been found to affect singing proficiency, such as tempo and the nature of the task (e.g., singing from memory versus imitation).

\section{The Neuronal Substrates of Singing}

The neuronal underpinnings of the human song system in healthy participants have been examined with neuroimaging techniques, such as PET and fMRI (Brown et al., 2004; Kleber et al., 2007; Perry et al., 1999; Zarate \& Zatorre, 2008). In some of these studies, singing was studied in non-musicians (e.g., Perry et al., 1999). Others focused either on amateur musicians (Brown et al., 2004), or on professional singers (Kleber et al., 2007). Moreover, different tasks were used (e.g., repeating a syllable on a tone, melody repetition, covert versus overt singing), which are typically associated with different brain activations (Brown et al., 2004). However, regardless of this variability, these studies uncovered a quite consistent functional network underlying singing, including motor and sensory areas as well as auditory-motor integration regions. Singing recruits regions of the primary motor cortex, such as the mouth region (Brown et al., 2004), and the larynx/phonation area, activated by adduction/ abduction and tension/relaxation of the vocal folds (Brown et al. 2008). Primary auditory cortex (i.e., the superior temporal gyrus, STG) is also engaged by vocal performance-for example, when repeating a single note (Perry et al., 1999), or singing more complex melodies (Brown et al., 2004; Kleber et al., 2007). A few other cortical areas are also systematically activated during vocal performance, such as the supplementary motor area (SMA), the anterior cingulate cortex (ACC) and the insula (Brown et al., 2004; Kleber et al., 2007; Perry et al., 1999; Zarate \& Zatorre, 2008). The SMA participates in high-level motor control, needed for efficient motor planning in sequence production. The ACC, involved in initiation of vocalisation, is implicated in both overt speech and singing (Paus, 2001; Perry et al., 1999). Finally, the anterior insula is associated with vocalisation processes, mostly articulation (Dronkers, 1996). These areas are indicated schematically in Figure 1 (for a detailed review, see Berkowska \& Dalla Bella, 2009b).

A few studies investigated how music lateralises to different hemispheres as compared to speech production (refer to Gordon et al., 2006, for a review). These studies reveal predominant right-hemisphere dominance in singing versus speaking (Ackermann \& Riecker, 2004; Callan et al., 2006; Jeffries et al., 2003; Riecker et al., 2000; Wildgruber et al., 1996). However, there is increasing evidence pointing to more bilateral involvement in singing than in speech production (Brown et al., 2006). For example, both right- and left-hemisphere anesthetisation interfere with singing 


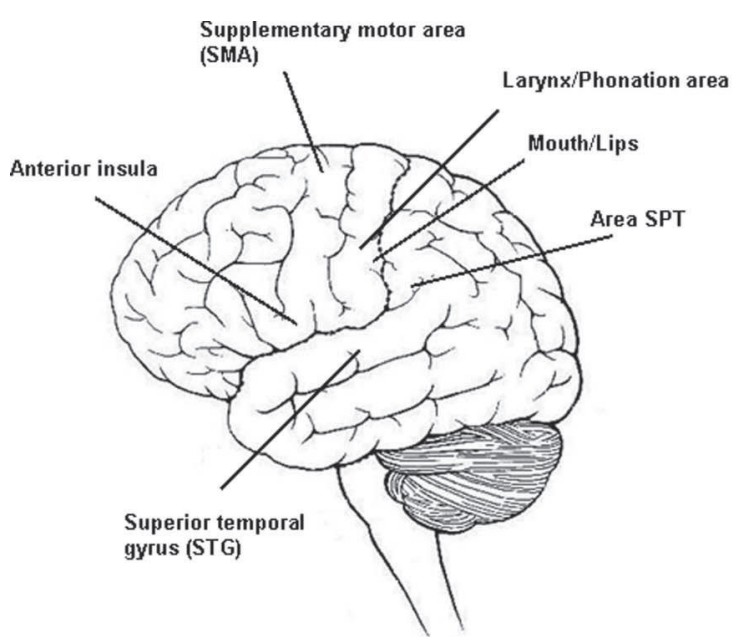

Figure 1 Areas of the brain associated with singing. Source: Berkowska \& Dalla Bella (2009b).

(Borchgrevink, 1980; Zatorre, 1984). Moreover, brain stimulation of the right frontal cortex via Transcranial Magnetic Stimulation disrupts singing in only a minority of individuals (Epstein et al., 1999).

In spite of these differences, however, there is a significant overlap of brain areas engaged by singing and speaking (Brown et al., 2006). In particular, certain regions (e.g., the inferior sensorimotor cortex and the superior temporal gyrus), activated both when singing and speaking (Gunji et al., 2007; Özdemir et al., 2006), are likely responsible for auditory-motor integration (Zarate \& Zatorre, 2008). This is a key process in monitoring pitch in vocal performance, which allows error correction during singing. More specifically, the area SPT (i.e., cortex of the dorsal Sylvian fissure at the parietal-temporal junction) is activated both during covert humming and covert speech (Hickok et al., 2003); this area is thought as functioning as a sensorimotor interface in speech production (Hickok \& Poeppel, 2007). Audio-vocal integration in singing was recently examined in an interesting study by Zarate and Zatorre (2008), in which non-musicians and experienced singers sang a single tone either with normal auditory feedback or with pitch-shifted feedback. Participants had to ignore the feedback or to compensate by pitch correction. Experienced singers, albeit more accurate in producing single pitches, recruited a very similar neural network to the one observed in non-musicians. In particular, this study suggests that the dorsal premotor cortex act as a basic auditory-motor interface. Other cortical regions such as the ACC and the auditory cortex would be more involved as vocal training and practice increase.

In sum, a complex neuronal network underpins singing, including sensory, motor and sensorimotor integration areas. These mechanisms can be disrupted by brain 


\section{S. Dalla Bella and M. Berkowska}

damage, thus leading to poor singing. An exhaustive review of impairments of sung performance consequent to brain damage is beyond the scope of this article (for reviews, see Berkowska \& Dalla Bella, 2009b; Gordon et al., 2006). However, a few comments are in order in relation to previous neuroimaging evidence in healthy individuals. The study of brain-damaged patients generally confirms the idea of righthemisphere dominance for pitch production, involving fronto-temporal regions (Alcock et al., 2000; Murayama, et al., 2004; Terao et al., 2006). However, in keeping with previously mentioned evidence, lesions in either of the two hemispheres can bring about poor singing (Kinsella et al., 1988; Prior et al, 1990), thus confirming that singing is unlikely to be a merely right-hemisphere function.

Moreover, it is worth noting that brain damage can selectively disrupt singing while leaving perception relatively intact (Confavreux et al., 1992; Schön et al., 2004). For example, Schön and colleagues reported the case of a tenor singer (IP) with right-hemisphere lesions distributed in the inferior frontal gyrus, posterior temporal lobe and inferior parietal lobe. IP exhibited expressive vocal amusia. His production of musical intervals was dramatically impaired. Nevertheless, his production of rhythm and contour was spared, as well as his music perception skills and language abilities. This suggests that pitch production and perception may be under the control of separate neuronal networks. This possibility is discussed further in the next section.

To summarise, neuroimaging evidence and lesion studies converge in indicating that there is not a brain centre for singing. Rather this activity recruits a complex neuronal network, involving several primary and secondary brain areas, which can be the target of brain damage, thus leading to poor singing. In the next section, we will turn to the examination of poor singing in the healthy non-musician population.

\section{Poor Singing in the General Population}

Another relevant source of evidence for understanding the mechanisms underlying proficient singing comes from the study of poor singers in the general population (i.e., often referred to as 'tone-deaf individuals). Indeed, in spite of the fact that occasional singers can typically sing in-tune and in-time, a few individuals have notorious difficulties in carrying a tune. Poor singing, observed approximately in 10$15 \%$ of the general population (Dalla Bella \& Berkowska, 2009; Dalla Bella et al., 2007; Pfordresher \& Brown, 2007), is often considered a hallmark of a more general lack of musicality, or tone deafness (Sloboda et al., 2005). The widespread term 'tone deafness' literally suggests that poor singing may be the consequence of deficient perception. Indeed, lack of musicality in the general population has been mostly associated with poor perceptual abilities - a condition referred to as 'congenital amusia' (Ayotte et al., 2002; Foxton et al., 2004; Peretz, 2001; Peretz et al., 2002; Peretz \& Hyde, 2003). Congenital amusia is a neurogenetic disorder affecting approximately 4\% of the population (Kalmus \& Fry, 1980; Peretz \& Hyde, 2003; Peretz et al., 2007). This condition can be uncovered by a battery of tests including 
pitch perception, rhythm perception and incidental memory tasks (i.e., the Montreal Battery of Evaluation of Amusia; Peretz et al., 2003). Congenital amusia is associated with brain anomalies in the right inferior frontal cortex (Hyde et al., 2006) and in the right auditory cortex (Hyde et al., 2007). Individuals with congenital amusia exhibit mostly impoverished pitch perception (Ayotte et al., 2002; Foxton et al., 2004; Hyde \& Peretz, 2004).

Fine perceptual monitoring of the vocal output (e.g., by allowing error correction guided by the auditory feedback) is likely to be key for accurate singing. This link between perception and performance was confirmed in a recent study (Dalla Bella et al., 2009), in which we asked congenital amusics to sing a highly familiar tune with lyrics from memory. Most, but not all, amusics were poor pitch singers (e.g., they inaccurately produced several intervals as compared with the notation and made contour errors). Yet, more than half sang in time. Since all amusics exhibited impaired pitch perception, these findings indicate that perception and performance are tightly coupled (but see below for exceptions).

However, perceptual deficits do not necessarily accompany poor singing. The observation that poor singing occurs more often (10-15\%) in the general population than congenital amusia (approximately 4\%) suggests that some individuals, despite normal perceptual abilities, may still be poor singers (Peretz \& Hyde, 2003). This dissociation between perception and performance is supported by a growing body of evidence (Bradshaw \& McHenry, 2005; Dalla Bella et al., 2007; Pfordresher \& Brown, 2007; Wise \& Sloboda, 2008). For example, in a recent study we described two occasional singers who, in spite of their markedly inaccurate production of pitch intervals (i.e., they deviated by more than one semitone from the notated intervals), normally detected pitch and time incongruities in unfamiliar melodies (Dalla Bella et al., 2007). Other examples of poor pitch singing with spared perception were reported by Pfordresher and Brown (2007) using a task in which participants imitated short novel melodies. Some $13 \%$ of the participants were classified as poor pitch singers (i.e., they transposed the pitches to be imitated by \pm 1 semitone). Yet, they performed as accurately as proficient singers in a pitch discrimination task (see also Wise \& Sloboda, 2008).

To summarise, deficient perception is not a necessary condition for poor singing. The opposite finding (i.e., impaired perception with spared production) is more striking. Loui et al. (2008) asked congenital amusics to imitate pitch and, in a second task, to judge if the second tone in a pair was higher or lower than the first. Congenital amusics were able to reproduce pitch direction (ascending or descending). Nevertheless, they could not detect pitch direction. More recently, we showed that this mismatch between perception and performance might not confine to pitch direction. We recently reported that two amusics with severely deficient pitch perception were able to sing with lyrics as proficiently as matched controls (Dalla Bella et al., 2009). The possibility that perception and action are less tightly coupled in congenital amusics is supported by recent evidence of abnormally reduced connectivity of the fasciculus arcuatus (i.e., a pathway connecting perception and 
action brain areas) in this population (Loui et al., 2009). Together, these results suggest that auditory perception and action, albeit in general tightly coupled, may be supported by separate neuronal networks (Griffiths, 2008).

The data reviewed so far show that there exist at least two categories of poor singers in the general population, with or without concurrent perceptual deficits. However, recent data reveal finer distinctions within these two categories, leading to a variety of singing 'phenotypes'. Patterns of poor singing were examined in a group of occasional singers who performed a battery of tests for the assessment of singing proficiency (Sung Performance Battery) including singing from memory and imitation tasks (Berkowska \& Dalla Bella, 2009; Dalla Bella \& Berkowska, 2009). Poor singers were in general less accurate on the pitch dimension than on the time dimension, in keeping with previous results (Dalla Bella et al., 2007, 2009). Some poor singers transposed pitch by more than four semitones (i.e., pitch 'transposers') without being inaccurate on the other dimensions, while others systematically deviated from the tempo of the melody to be imitated (i.e., tempo 'transposers'). Others were inaccurate in producing interval size (i.e., poor pitch interval singers), deviating by more than one semitone on average from the notated intervals, with little transposition. Finally, a minority inaccurately produced note relative durations (i.e., poor duration singers). In sum, inaccurate singing can concern specific components of the general ability to sing, dedicated to relative or absolute aspects of pitch and time processing. These components may recruit partially independent neuronal networks - a possibility that deserves further enquiry.

The diversity of poor singing phenotypes can be accounted for by various factors (e.g., poor perception, poor motor planning and execution, poor auditory-motor integration; see Pfordresher \& Brown, 2007). A simplified schema of the components of the human song system, which can be malfunctioning in poor singers, is provided in Figure 2. Singing familiar melodies from memory requires the retrieval of pitch

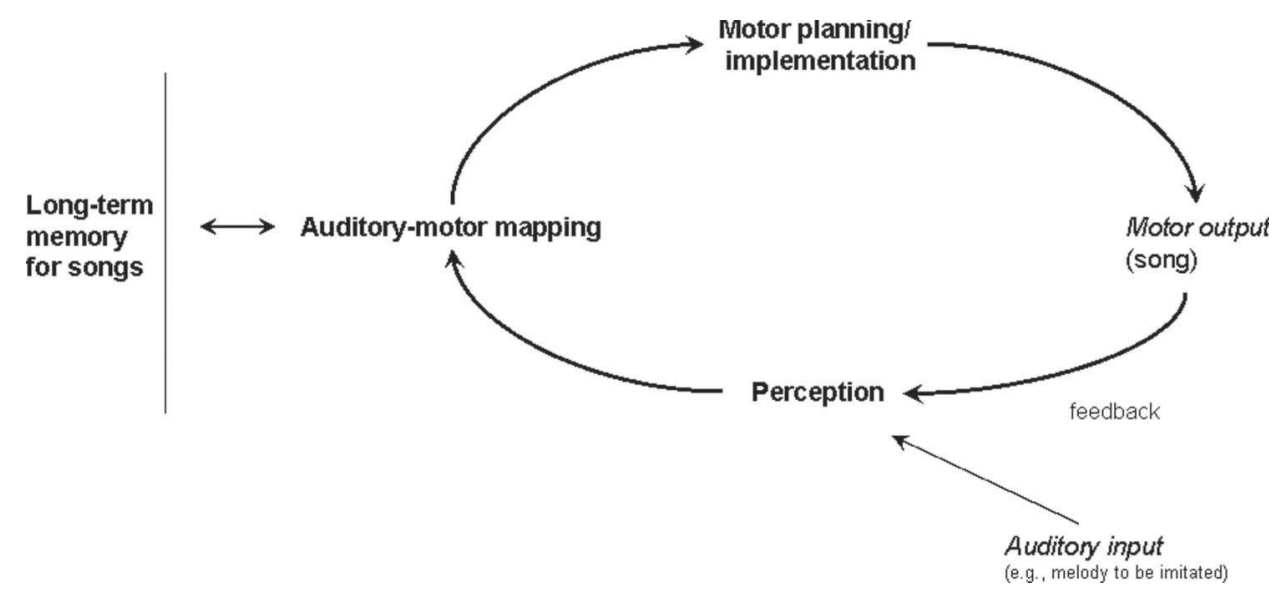

Figure 2 Components of the human vocal system that might be malfunctioning in poor singers. 
and temporal information from long-term memory and fine motor planning/ implementation. The perceptual analysis of the vocal output in comparison with the intended melody is also a key process in error correction during singing. Similar mechanisms are probably engaged in melody imitation, where the target melody to be imitated is first perceived, stored in short-term memory and then the stored pitches are mapped into motor gestures. As before, a feedback mechanism allows the singer to monitor his or her ongoing performance, and to correct errors if needed. The disruption of any of these mechanisms will result in poor singing.

\section{Concluding Remarks}

In this article, evidence regarding singing proficiency in occasional singers was reviewed. The majority of occasional singers can sing proficiently. Studies into the neurosciences of music indicate that proficient singing does not result from the activity of a single 'song area' in the brain. Rather, a complex neuronal network underlying perceptual, motor, auditory-motor integration and memory regions underpins the human vocal system. Identifying and describing the components of these neuronal networks and their connectivity is essential for understanding normal singing in non-musicians and expert musicians. In addition, in future it will allow specifying the effect of musical/vocal experience on singing proficiency, and on its neuronal correlates (e.g., which specific components are affected by training, and under which conditions). Finally, this knowledge is contributing to an account of individual differences in the general population (e.g., why some individuals from the very beginning are poor singers while others are proficient), up to providing some 'explanation' for tone deafness (Loui et al., 2009). Indeed, when some components of this network are disrupted by brain damage or their development brought to a halt by neurogenetic disorders (e.g., congenital amusia), the song system is malfunctioning, thus leading to poor singing. In turn, poor-singing phenotypes can be very specific and reflect the properties of the brain network being disrupted.

For musicians and music educators, knowing which brain mechanisms are responsible for proficient singing may be very valuable for devising the appropriate pedagogical strategy—-for instance, with children exhibiting inaccurate singing. Knowing that some components of the song system are selectively less functional than others may lead one to devise and practice exercises tapping this particular skill, thereby leading to improve singing proficiency. Finally, this knowledge will ultimately provide useful information for understanding the beneficial effect of vocal performance in rehabilitation (Götell et al., 2003; Schlaug et al., 2008; Tamplin, 2008).

\section{Acknowledgments}

Dalla Bella's research was supported by a research grant from the Grammy Foundation. We thank Lauren Stewart and the anonymous reviewers for insightful comments on an earlier version of the manuscript. 


\section{References}

Ackermann, H. \& Riecker, A. (2004). The contribution of the insula to motor aspects of speech production: A review and hypothesis. Brain and Language, 89, 320-328.

Alcock, K. J. et al. (2000). Pitch and timing abilities in adult left-hemisphere dysphasic and righthemisphere damaged subjects. Brain and Language, 75, 47-65.

Amir, O., Amir, N. \& Kishon-Rabin, L. (2003). The effect of superior auditory skills on vocal accuracy. Journal of the Acoustical Society of America, 113, 1102-1108.

Ayotte, J., Peretz, I. \& Hyde, K. (2002). Congenital amusia: A group study of adults afflicted with a music-specific disorder. Brain, 125, 238-251.

Bergeson, T. R. \& Trehub, S. E. (2002). Absolute pitch and tempo in mothers' songs to infants. Psychological Science, 13, 72-75.

Berkowska, M. \& Dalla Bella, S. (2009a). Reducing linguistic information enhances singing proficiency in occasional singers. Annals of the New York Academy of Sciences, 1169, 108-111.

Berkowska, M. \& Dalla Bella, S. (2009b). Acquired and congenital disorders of sung performance: A review. Advances in Cognitive Psychology, 5, 69-83.

Borchgrevink, H. M. (1980). Cerebral lateralization of speech and singing after intracarotid amytal injection. In M. T. Sarno \& O. Hook (Eds.), Aphasia: Assessment and treatment (pp. 186191). Stockholm: Almqvist \& Wiksell.

Bradshaw, E. \& McHenry, M. A. (2005). Pitch discrimination and pitch-matching abilities of adults who sing inaccurately. Journal of Voice, 14, 431-439.

Brown, S., Martinez, M. J. \& Parsons, L. M. (2006). Music and language side by side in the brain: A PET study of the generation of melodies and sentences. European Journal of Neuroscience, 23, 2791-2803.

Brown, S., Ngan, E. \& Liotti, M. (2008). A larynx area in the human motor cortex. Cerebral Cortex, $18,837-845$.

Brown, S. et al. (2004). The song system of the human brain. Cognitive Brain Research, 20, 363-375.

Callan, D. E. et al. (2006). Song and speech: Brain regions involved with perception and covert production. Neuroimage, 31, 1327-1342.

Confavreux, C. et al. (1992). Progressive amusia and aprosody. Archives in Neurology, 49, 971-976.

Cuddy, L. L. et al. (2005). Musical difficulties are rare: A study of 'tone deafness' among university students. Annals of the New York Academy of Sciences, 1060, 311-324.

Dalla Bella, S. \& Berkowska, M. (2009). Singing proficiency in the majority: Normality and 'phenotypes' of poor singing. Annals of the New York Academy of Sciences, 1169, 99-107.

Dalla Bella, S., Giguère, J.-F. \& Peretz, I. (2007). Singing proficiency in the general population. Journal of the Acoustical Society of America, 121, 1182-1189.

Dalla Bella, S., Giguère, J.-F. \& Peretz, I. (2009). Singing in congenital amusia. Journal of the Acoustical Society of America, 126, 414-424.

Dowling, W. J. (1999). The development of music perception and cognition. In D. Deutsch (Ed.), The psychology of music. (pp. 603-625). San Diego, CA: Academic Press.

Dowling, W. J. \& Harwood, D. (1986). Music cognition. New York: Academic Press.

Dronkers, N. F. (1996). A new brain region for coordinating speech articulation. Nature, 384, 159161.

Epstein, C. M. et al. (1999). Localization and characterization of speech arrest during transcranial magnetic stimulation. Clinical Neurophysiology, 110, 1073-1079.

Foxton, J. M. et al. (2004). Characterization of deficits in pitch perception underlying 'tone deafness'. Brain, 127, 801-810.

Gordon, R. L., Racette, A. \& Schön, D. (2006). Sensory-motor networks in singing and speaking: A comparative approach. In E. Altenmüller, M. Wiesendanger \& J. Kesselring (Eds.), Music, motor control and the brain (pp. 205-222). Oxford: Oxford University Press. 
Götell, E., Brown, S. \& Ekman, S.-L. (2003). Influence of caregiver singing and background music on posture, movement, and sensory awareness in dementia care. International Psychogeriatrics, 15, 411-430.

Griffiths, T. D. (2008). Sensory systems: Auditory action streams? Current Biology, 18, R387-388.

Gunji, A. et al. (2007). Rhythmic brain activities related to singing in humans. Neuroimage, 34, 426434.

Halpern, A. R. (1989). Memory for the absolute pitch of familiar songs. Memory \& Cognition, 17, 572-581.

Hébert, S. et al. (2003). Revisiting the dissociation between singing and speaking in expressive aphasia. Brain, 126, 1838-1850.

Hickok, G. \& Poeppel, D. (2007). The cortical organization of speech processing. Nature Reviews Neuroscience, 8, 393-402.

Hickok, G. et al. (2003). Auditory-motor interaction revealed by fMRI: Speech, music and working memory in area Spt. Journal of Cognitive Neuroscience, 15, 673-682.

Himonides, E. \& Welch, G. (2006). A large-scale survey regarding listeners' tastes to sung performances. Paper presented at the Ninth International Conference of Music Perception and Cognition, Bologna, Italy.

Hyde, K. L. \& Peretz, I. (2004). Brains that are out of tune but in time. Psychological Science, 15, 356-360.

Hyde, K. L. et al. (2006). Morphometry of the amusic brain: A two-site study. Brain, 129, 25622570.

Hyde, K. L. et al. (2007). Cortical thickness in congenital amusia: When less is better than more. Journal of Neuroscience, 27, 13028-13032.

Jeffries, K. J., Fritz, J. B. \& Braun, A. R. (2003). Words in melody: $\mathrm{An}_{2}{ }^{15} \mathrm{O}$ PET study of brain activation during singing and speaking. Neuroreport, 15, 749-754.

Kalmus, H. \& Fry, D. B. (1980). On tune deafness (dysmelodia): Frequency, development, genetics and musical background. Annals of Human Genetics, 43, 369-382.

Kinsella, G., Prior, M. R. \& Murray, G. (1988). Singing ability after right- and left-sided brain damage: A research note. Cortex, 24, 165-169.

Kleber, B. et al. (2007). Overt and imagined singing of an Italian aria. Neuroimage, 36, 889-900.

Levitin, D. J. (1994). Absolute memory for musical pitch: Evidence from the production of learned melodies. Perception \& Psychophysics, 56, 414-423.

Levitin, D. J. \& Cook, P. R. (1996). Memory for musical tempo: Additional evidence that auditory memory is absolute. Perception \& Psychophysics, 58, 927-935.

Loui, P., Alsop, D. \& Schlaug, G. (2009). Tone deafness: A new disconnection syndrome? Journal of Neuroscience, 29, 10215-10220.

Loui, P. et al. (2008). Action-perception mismatch in tone-deafness. Current Biology, 18, R331-332.

Mithen, S. (2006). The singing Neanderthals. Cambridge, MA: Harvard University Press.

Murayama, J. et al. (2004). Impaired pitch production and preserved rhythm production in a right brain-damaged patient with amusia. Brain and Cognition, 56, 36-42.

Murry, T. (1990). Pitch-matching accuracy in singers and nonsingers. Journal of Voice, 4, 317321.

Murry, T. \& Zwiner, P. (1991). Pitch matching ability of experienced and inexperienced singers. Journal of Voice, 5, 197-202.

Ostwald, P. F. (1973). Musical behavior in early childhood. Developmental Medicine and Child Neurology, 15, 367-375.

Özdemir, E., Norton, A. \& Schlaug, G. (2006). Shared and distinct neural correlates of singing and speaking. NeuroImage, 33, 628-635.

Papoušek, H. (1996). Musicality in infancy research: Biological and cultural origins of early musicality. In I. Deliège \& J. Sloboda (Eds.), Musical beginnings (pp. 37-55). Oxford: Oxford University Press. 


\section{S. Dalla Bella and M. Berkowska}

Paus, T. (2001). Primate anterior cingulate cortex: Where motor control, drive and cognition interact. Nature Reviews Neuroscience, 2, 417-424.

Peretz, I. (2001). Brain specialization for music: New evidence from congenital amusia. Annals of the New York Academy of Sciences, 930, 189-192.

Peretz, I., Champod, S., \& Hyde, K. (2003). Varieties of musical disorders: The Montreal Battery of Evaluation of Amusia. Annals of the New York Academy of Sciences, 999, 58-75.

Peretz, I., Cummings, S. \& Dubé, M.-P. (2007). The genetics of congenital amusia (tone deafness): A family-aggregation study. American Journal of Human Genetics, 81, 582-588.

Peretz, I. \& Hyde, K. (2003). What is specific to music processing? Insights from congenital amusia. Trends in Cognitive Sciences, 7, 362-367.

Peretz, I. et al. (2002). Congenital amusia: A disorder of fine-grained pitch discrimination. Neuron, 33, 185-191.

Perry, D. W. et al. (1999). Localization of cerebral activity during simple singing. Neuroreport, 10, 3979-3984.

Pfordresher, P. Q. \& Brown, S. (2007). Poor-pitch singing in the absence of 'tone-deafness'. Music Perception, 25, 95-115.

Prior, M., Kinsella, G. \& Giese, J. (1990). Assessment of musical processing in brain-damaged patients: Implications for laterality of music. Journal of Clinical and Experimental Neuropsychology, 12, 301-312.

Racette, A., Bard, C. \& Peretz, I. (2006). Making non-fluent aphasics speak: Sing along! Brain, 129, 2571-2584.

Riecker, A. et al. (2000). Opposite hemispheric lateralization effects during speaking and singing at motor cortex, insula and cerebellum. Neuroreport, 11, 1997-2000.

Schlaug, G., Marchina, S. \& Norton, A. (2008). From singing to speaking: Why singing may lead to recovery of expressive language function in patients with Broca's aphasia. Music Perception, $25,315-323$.

Schön, D. et al. (2004). A selective deficit in the production of exact musical intervals following right-hemisphere damage. Cognitive Neuropsychology, 21, 773-784.

Sloboda, J. A., Wise, K. J. \& Peretz, I. (2005). Quantifying tone deafness in the general population. Annals of the New York Academy of Sciences, 1060, 255-261.

Sundberg, J. (1987). The science of the singing voice. DeKalb, IL: Northern Illinois University Press.

Sundberg, J. (1999). The perception of singing. In D. Deutsch (Ed.), The psychology of music (pp. 171-214). San Diego, CA: Academic Press.

Tamplin, J. (2008). A pilot study into the effect of vocal exercises and singing on dysarthric speech. Neurorehabilitation, 23, 207-216.

Terao, Y. et al. (2006). Vocal amusia in a professional tango singer due to a right superior temporal cortex infarction. Neuropsychologia, 44, 479-488.

Ternstrom, S., Sundberg, J. \& Collden, A. (1988). Articulatory F0 perturbations and auditory feedback. Journal of Speech and Hearing Research, 31, 187-192.

Trehub, S. E. (2009). Music lessons from infants. In S. Hallam, I. Cross \& M. Thaut (Eds.), The Oxford handbook of music psychology (pp. 229-234). Oxford: Oxford University Press.

Trehub, S. E. \& Trainor, L. J. (1999). Singing to infants: Lullabies and play songs. Advances in Infancy Research, 12, 43-77.

Vurma, A. \& Ross, J. (2006). Production and perception of musical intervals. Music Perception, 23, 331-344.

Wallin, N. L., Merker, B. \& Brown, S. (2000). The origins of music. Cambridge, MA: MIT Press.

Welch, G. F. (2005). Singing as communication. In D. Miell, R. MacDonald \& D. Hargreaves (Eds.), Musical communication (pp. 239-259). New York: Oxford University Press.

Welch, G. F. (2006). Singing and vocal development. In G. McPherson (Ed.), The child as musician: A handbook of musical development (pp. 311-329). New York: Oxford University Press. 
Wermke, K., Leising, D. \& Stellzig-Eisenhauer, A. (2007). Relation of melody complexity in infants' cries to language outcome in the second year of life: A longitudinal study. Clinical Linguistics \& Phonetics, 21, 961-973.

Wildgruber, D. et al. (1996). Functional lateralization of speech production at primary motor cortex: A fMRI study. Neuroreport, 7, 2791-2795.

Wise, K. J. \& Sloboda, J. A. (2008). Establishing an empirical profile of self-defined 'tone deafness': Perception, singing performance and self-assessment. Musicae Scientiae, 12, 3-23.

Zarate, J. M. \& Zatorre, R. J. (2008). Experience-related neural substrates involved in vocal pitch regulation during singing. NeuroImage, 40, 1871-1887.

Zatorre, R. J. (1984). Musical perception and cerebral function: A critical review. Music Perception, 2, 196-221. 